\title{
Ações de Educação em Saúde Para Idosos na Atenção Básica: Revisão de Literatura
}

\author{
Francisco Jaime Rodrigues de Lima Filho ${ }^{1}$, Ingrid Grangeiro Bringel Silva ${ }^{2}$, \\ Nalva Kelly Gomes de Lima ${ }^{3}$, Michellane de Miranda Pontes ${ }^{4}$, \\ Ylkiany Pereira de Souza ${ }^{5}$, Thayse Wilma Nogueira de Oliveira ${ }^{6}$
}

\begin{abstract}
RESUMO
O presente artigo tem como objetivo identificar na literatura evidências científicas acerca da utilização da educação em saúde na atenção básica como promotora de um envelhecimento saudável. Trata-se de uma revisão bibliográfica, desenvolvida nas bases de dados Literatura Latino-Americana em Ciências da Saúde (Lilacs), Base de Dados de Enfermagem (BDENF) e na biblioteca eletrônica Scientific Electronic Library On-line (SciELO), aplicando os descritores: Envelhecimento, Educação em saúde e Atenção básica. Os descritores foram associados nas bases de dados utilizando o operador booleano "and". Como critérios de inclusão, elegeram-se trabalhos originais, publicados entre os anos de 2008 e 2017, no intuito de alcançar o maior número de evidências científicas sobre o tema, nos idiomas inglês, português e espanhol, disponíveis on-line gratuitamente. Verificou-se que as ações educativas são compreendidas como necessárias para a atenção ao idoso, embora os profissionais apontem dificuldades para a realização das mesmas, como falta de preparo para tratar da temática, descaso da gestão e falta de insumos. Conclui-se que ações educativas têm potencial para garantir melhoria na qualidade de vida dos idosos, tanto nos aspectos biológicos quanto nos psicossociais.

Palavras-chave: Envelhecimento. Educação em saúde. Atenção básica.

\section{HEALTH EDUCATION ACTIONS FOR ELDERLY PEOPLE IN BASIC ATTENTION: LITERATURE REVIEW}

\section{ABSTRACT}

The present article aims to identify in the literature scientific evidence about the use of health education in primary care as a promoter of healthy aging. This is a bibliographical review, developed in the Latin American Literature in Health Sciences (Lilacs), Nursing Database (BDENF) and Scientific Electronic Library Online (SciELO) electronic databases, applying the following descriptors: Aging, Health Education and Primary Care. The descriptors were associated in the databases using the boolean operator "and". As inclusion criteria, we chose: original papers, published between 2008 and 2017, in order to reach the highest scientific evidence on the subject, in English, Portuguese and Spanish, available online free of charge. It was verified that the educational actions are understood as necessary for attention to the elderly, although the professionals point out difficulties to perform them, such as: lack of preparation to deal with the issue, lack of management and lack of inputs. It is concluded that educational actions have the potential to guarantee improvement in the quality of life of the elderly, both in biological and psychosocial aspects.
\end{abstract}

Keywords: Aging. Health education. Basic attention

Recebido em: 12/11/2017

Aceito em: $13 / 7 / 2018$

\footnotetext{
Enfermeiro. Residente em Saúde Coletiva pelo Instituto Aggeu Magalhães - Fiocruz/PE. franciscojaime8@gmail.com

2 Enfermeira. Mestranda em Enfermagem pela Universidade Regional do Cariri. ingrid_gbringel@hotmail.com

${ }^{3}$ Enfermeira. Residente em Enfermagem em Cardiologia pela Universidade de Pernambuco. nalvakellygomes@gmail.com

${ }^{4}$ Enfermeira. Residente em Enfermagem em Cardiologia pela Universidade de Pernambuco. michellane_miranda@hotmail.com

5 Enfermeira. Residente em Urgência e Emergência pela Universidade de Pernambuco. ylkiany@hotmail.com

${ }^{6}$ Nutricionista. Residente no Programa de Residência em Nutrição Clínica do Hospital dos Servidores do Estado de Pernambuco (Coremu/Ufpe). thaysewilma@yahoo.com.br
} 


\section{INTRODUÇÃO}

O envelhecimento configura-se uma experiência permeada por modificações complexas na vida dos indivíduos. No que diz respeito aos aspectos biológicos, esse processo implica o acúmulo de danos a nível celular e molecular, acarretando em diminuição de reservas fisiológicas, aumento do risco de adquirir doenças e declínio da capacidade geral dos sujeitos. Salienta-se que tais características não são iguais para todos os indivíduos, sendo associada, de maneira vaga, a idade das pessoas em anos (ORGANIZAÇÃO..., 2015).

Por outro lado, verifica-se que, para além das perdas biológicas, o processo associado ao envelhecimento também implica modificações no tocante à vida em sociedade, com a troca progressiva de papéis sociais pelos indivíduos. Relacionado a isso, percebe-se a modificação de objetivos e perspectivas dos idosos, guiadas pela adaptação contínua às perdas vivenciadas ou ao próprio amadurecimento psicológico associado ao avançar da idade (BALTES; FREUND; LI, 2005). Salienta-se que, de acordo com a Política Nacional de Saúde da Pessoa Idosa, consideram-se idosos os indivíduos com 60 anos ou mais (BRASIL, 2006).

No que concerne aos aspectos demográficos, o Brasil apresenta-se como um dos países que demonstram um acelerado processo de modificação da sua estrutura etária, relacionado principalmente às quedas no nível de fecundidade e aumento da expectativa de vida da população. A pirâmide etária nacional tem apresentado diminuição de sua base, representada por crianças e indivíduos jovens, e um consequente aumento do ápice piramidal associado à população idosa. Se em meados da década de 50 do século 20 , a taxa de fecundidade era de aproximadamente seis filhos por mulher, esse número encontra-se próximo a dois filhos atualmente. Nesse sentido, verifica-se a necessidade de orientação das políticas sociais para o cuidado e atenção a esse estrato populacional (INSTITUTO..., 2015).

No tocante aos aspectos relacionados à morbidade nessa faixa etária, verificou-se, de acordo com a literatura, predominância de Doenças Crônicas Não Transmissíveis (DCNT), como Diabetes Mellitus, Hipertensão Arterial Sistêmica, Osteoporose, Ansiedade e Doenças Cardiovasculares (PEREIRA; NOGUEIRA; SILVA, 2015). Em relação às características da mortalidade dessa população, percebe-se que as principais causas de óbito estão associadas a doenças do apareIho circulatório, seguidas das neoplasias e doenças do aparelho respiratório (CARVALHO et al., 2014; OLIVEIRA; MEDEIROS; LIMA, 2015; SILVA, 2012).
Evidencia-se, desse modo, a necessidade de orientar esforços para a criação de estratégias que influenciem o envelhecimento ativo e saudável, no intuito de incentivar a adesão a hábitos de vida saudáveis. Nesse cenário, percebe-se que a atenção básica possui potencialidades referentes ao cuidado direcionado a esses usuários, posto que desenvolve a atenção em uma perspectiva territorial com ênfase em atividades relacionadas à prevenção e à promoção da saúde. Os profissionais da atenção básica dispõem de tecnologias capazes de garantir melhorias na qualidade de vida dos indivíduos, pautadas em métodos que levam em consideração o vínculo e as relações estabelecidas entre profissionais e usuários, como a educação em saúde. Atividades educativas podem garantir a aquisição de conhecimentos e habilidades para a adoção de estilos de vida saudáveis, por meio do empoderamento dos sujeitos acerca de sua saúde (BRASIL, 2006).

Por sua vez, a Educação em Saúde pode ser entendida como um processo que visa à utilização de um pensamento crítico para ler a realidade. Nesse sentido, homens e mulheres podem dispor de recursos para intervir e transformar as suas situações de saúde, alcançando-a enquanto um direito social conquistado (MAROSINI; FONSECA; PEREIRA, 2008).

O Ministério da Saúde adota como definição para educação em saúde o processo de construção de conhecimentos pela população sobre saúde, no intuito de garantir a autonomia dos sujeitos ante o seu processo saúde-doença-cuidado. Salienta-se que essas iniciativas também devem ser orientadas para potencialização do controle social em saúde, garantindo a melhoria das políticas públicas e dos serviços assistenciais (BRASIL, 2012).

Os processos educativos devem estar orientados para a preservação da autonomia dos sujeitos e para a valorização dos seus conhecimentos e necessidades, visando a garantir melhorias na qualidade de vida dos mesmos. Todas essas características devem basear-se numa perspectiva dialógica, de aproximação com a população, permitindo o intercâmbio de saberes e conhecimentos entre eles e os profissionais (MACIEL, 2009; FALKENBERG et al., 2014).

Pode-se considerar a educação uma importante ferramenta para a promoção da saúde, uma vez que mostra-se valiosa quando orientada para a prevenção de doenças, assim como para a reabilitação dos indivíduos. Salienta-se que a mesma ainda pode ser tida como um veículo para a transformação social, tendo em vista que pode contribuir com um melhor entendimento a respeito da cidadania, responsabilidade so- 
cial e a consequente formação de sujeitos capazes de multiplicar as informações adquiridas nesse processo para os seus pares (FEIJÃO; GALVÃO, 2007).

Desse modo, identificou-se a importância de verificar na literatura evidências científicas que abordem essa temática, no intuito de investigar qual o estado da arte sobre a mesma, assim como utilizar tais conhecimentos para orientar as práticas de saúde na realidade clínica, auxiliando os profissionais nas tomadas de decisão acerca de práticas de saúde que não sejam somente pensadas sob uma perspectiva centrada na doença e pouco dialógica. Desse modo, indaga-se: Quais ações de educação em saúde têm sido direcionadas para a população idosa na atenção básica? Como elas se processam nesse nível de atenção?

Nesse sentido, o presente estudo tem como objetivo identificar na literatura evidências científicas acerca da utilização da educação em saúde na atenção básica como promotora de um envelhecimento saudável.

\section{MÉTODO}

Trata-se de uma revisão bibliográfica da literatura desenvolvida entre os meses de setembro e novembro de 2017. Para guiar a estruturação do presente artigo utilizou-se a seguinte questão norteadora: "O que tem sido produzido e evidenciado na literatura sobre a utilização da educação em saúde na atenção básica como promotora de um envelhecimento saudável?"

Como fontes de informação utilizaram-se as bases de dados Literatura Latino-Americana em Ciências da Saúde (Lilacs), Base de Dados de Enfermagem (BDENF) e a biblioteca eletrônica Scientific Electronic Library Online (SciELO). Para a realização das buscas usaram-se Descritores em Ciências da Saúde (Decs) selecionados de acordo com elementos da questão norteadora, sendo eles: Envelhecimento, Educação em Saúde e Atenção básica. Os descritores de assunto foram associados utilizando-se o operador booleano "AND". A estratégia nas bases de dados com os Decs e o operador booleano configurou-se da seguinte maneira: envelhecimento and "educação em saúde" e "envelhecimento and atenção básica".

Como fatores de inclusão elegeram-se: trabalhos originais (artigo original, teses, dissertações e monografias) publicados entre os anos de $2008 \mathrm{e}$ 2017, no intuito de garantir o maior número possível de evidências científicas nos últimos 10 anos sobre o tema, nos idiomas inglês, português e espanhol, disponíveis on-line gratuitamente. A seleção dos artigos deu-se pela leitura sistemática dos mesmos a partir da ordem de filtragem: leitura de títulos, leitura de resumos e leitura de trabalhos na íntegra. Para a coleta de dados utilizou-se o protocolo para leitura de artigos, desenvolvido por Ursi (2005), permitindo coletar informações pertinentes aos autores dos artigos, seus objetivos, metodologia e resultados. Os resultados do presente estudo foram organizados em figuras e tabelas e sua apresentação estruturada de forma narrativa em categorias temáticas.

\section{RESULTADOS}

A partir das buscas nas bases de dados foram encontrados 300 trabalhos, dos quais, ao passarem pela fase de leitura crítica, com avaliação dos fatores de inclusão, 16 foram selecionados para compor a amostra do presente artigo. Na Figura 1 observa-se o fluxograma de busca nas bases de dados.

Figura 1 - Fluxograma de estudos selecionados

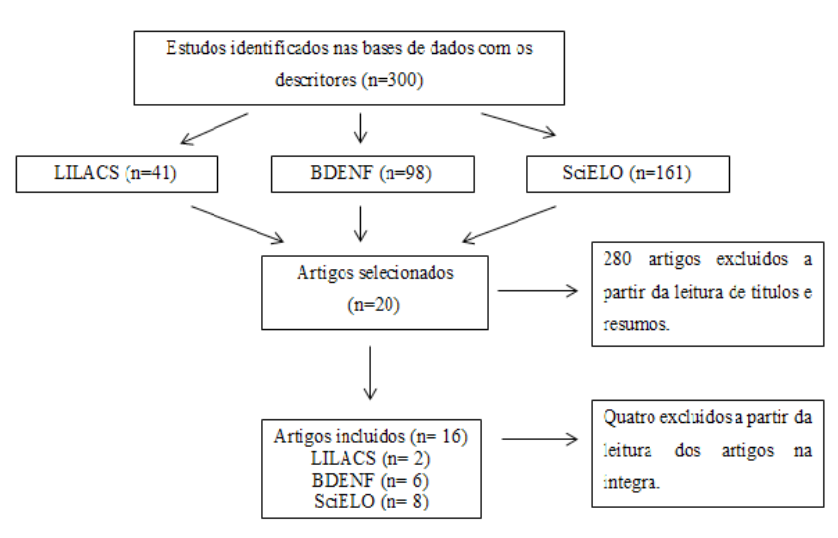

Dentre os estudos selecionados percebe-se que a maioria foi publicado no ano de 2010 (31,25\%), seguido do ano de 2013 (25\%). Verifica-se que a abordagem qualitativa nos estudos esteve mais presente e o idioma mais prevalente foi o português. Na tabela a seguir verificam-se as informações detalhadas de cada publicação.

A seguir apresenta-se a síntese dos estudos incluídos. Os mesmos foram organizados em categorias temáticas, sendo elas: percepção dos idosos sobre as ações de educação em saúde; percepção dos profissionais sobre as ações de educação em saúde; estratégias de educação em saúde para idosos; e avaliação das ações de educação em saúde na qualidade de vida de idosos.

\section{Percepção dos idosos participantes} de grupos de educação em saúde

Em estudo desenvolvido por Ferreira et al. (2015), verifica-se que idosos que fazem parte de grupos de promoção da saúde avaliaram sua qualidade 
Tabela 1 - Descrição dos trabalhos selecionados segundo autoria, ano de publicação, local de indexação, local do estudo, objetivo, método e idiomas disponíveis

\begin{tabular}{|c|c|c|c|c|}
\hline $\begin{array}{l}\text { Autoria, ano de } \\
\text { publicação e lo- } \\
\text { cal de indexação }\end{array}$ & $\begin{array}{l}\text { Local do } \\
\text { estudo }\end{array}$ & Objetivo & Método & $\begin{array}{l}\text { Idiomas } \\
\text { disponíveis }\end{array}$ \\
\hline $\begin{array}{l}\text { González (2008) } \\
\text { SciELO }\end{array}$ & $\begin{array}{l}\text { Rio de Ja- } \\
\text { neiro }\end{array}$ & $\begin{array}{l}\text { Analisar a incorporação das premissas da promoção da saúde no cuidado } \\
\text { a pessoas portadoras de hipertensão arterial em um serviço de atenção } \\
\text { primária à saúde. }\end{array}$ & $\begin{array}{l}\text { Pesquisa qualita- } \\
\text { tiva. Análise de } \\
\text { Conteúdo. }\end{array}$ & Português \\
\hline $\begin{array}{l}\text { Cruz; Martins } \\
\text { (2010) } \\
\text { BDENF }\end{array}$ & $\begin{array}{l}\text { Várzea Ale- } \\
\text { gre - CE }\end{array}$ & $\begin{array}{l}\text { Conhecer a percepção dos/as agentes comunitário/as de saúde sobre sua } \\
\text { atuação na promoção da saúde do idoso. }\end{array}$ & $\begin{array}{l}\text { Pesquisa qualita- } \\
\text { tiva, com aborda- } \\
\text { gem descritivo-ex- } \\
\text { ploratória. }\end{array}$ & Português \\
\hline $\begin{array}{l}\text { Araújo; Barbosa } \\
\text { (2010) } \\
\text { BDENF }\end{array}$ & $\begin{array}{l}\text { Aparecida } \\
\text { de Goiânia } \\
\text { - GO }\end{array}$ & Discutir a relação dos profissionais de saúde da família com os idosos. & $\begin{array}{l}\text { Pesquisa qualita- } \\
\text { tiva. }\end{array}$ & Português \\
\hline $\begin{array}{l}\text { Baldissera; Bue- } \\
\text { no (2010) } \\
\text { BDENF }\end{array}$ & Paraná & $\begin{array}{l}\text { Desenvolver e avaliar estratégias de educação para a saúde baseada na } \\
\text { pedagogia crítico-social, partindo da representação social da sexualidade } \\
\text { junto as mulheres portadoras de HAS participantes de um grupo de encon- } \\
\text { tro de um centro de saúde no Noroeste do Estado do Paraná-Brasil. }\end{array}$ & $\begin{array}{l}\text { Pesquisa qualitati- } \\
\text { va, do tipo pesqui- } \\
\text { sa-ação. }\end{array}$ & Português \\
\hline $\begin{array}{l}\text { Ferreira et al. } \\
\quad(2010) \\
\text { SciELO }\end{array}$ & Goiás & $\begin{array}{l}\text { O estudo tem como objetivo discutir a relação dos profissionais de saúde } \\
\text { da família com os idosos. }\end{array}$ & $\begin{array}{l}\text { Pesquisa quanti- } \\
\text { tativa }\end{array}$ & $\begin{array}{l}\text { Português } \\
\text { Inglês } \\
\text { Espanhol }\end{array}$ \\
\hline $\begin{array}{l}\text { Araújo (2010) } \\
\text { BDENF }\end{array}$ & $\begin{array}{l}\text { Campina } \\
\text { Grande - } \\
\text { PB }\end{array}$ & $\begin{array}{l}\text { Conhecer as experiências dos profissionais que trabalham com educação } \\
\text { em saúde voltada para idosos na atenção básica. }\end{array}$ & $\begin{array}{l}\text { Pesquisa qualita- } \\
\text { tiva, utilizando o } \\
\text { método da histó- } \\
\text { ria oral temática. }\end{array}$ & Português \\
\hline $\begin{array}{l}\text { Barros; Maia; } \\
\text { Pagliuca (2011) } \\
\text { BDENF }\end{array}$ & $\begin{array}{l}\text { Caririaçu } \\
-\mathrm{CE}\end{array}$ & $\begin{array}{l}\text { Apresentar o perfil dos profissionais da Estratégia de Saúde da Família, } \\
\text { identificando ações prestadas, facilidades e dificuldades enfrentadas na } \\
\text { assistência ao idoso. }\end{array}$ & $\begin{array}{l}\text { Pesquisa qualita- } \\
\text { tiva com aborda- } \\
\text { gem descritiva. }\end{array}$ & Português \\
\hline $\begin{array}{l}\text { Baldissera; Bue- } \\
\text { no (2012) } \\
\quad \text { Lilacs }\end{array}$ & Paraná & $\begin{array}{l}\text { Realizar, por meio de uma pesquisa-ação a Educação para a Saúde junto } \\
\text { a um grupo de portadores de HAS baseada na pedagogia crítico-social, } \\
\text { partindo da percepção dos participantes quanto ao lazer, desenvolvendo } \\
\text { atividades educativas e, posteriormente, avaliando a opinião dos envolvi- } \\
\text { dos quanto ao impacto para a vida e para a saúde mental. }\end{array}$ & Pesquisa-ação & Português \\
\hline $\begin{array}{l}\text { Araújo et al. } \\
\text { (2013) } \\
\text { Lilacs }\end{array}$ & $\begin{array}{l}\text { Campina } \\
\text { Grande - } \\
\text { PB }\end{array}$ & $\begin{array}{l}\text { Conhecer as experiências dos profissionais de saúde que trabalham com } \\
\text { educação em saúde voltada para idosos na Estratégia Saúde da Família e } \\
\text { identificar as ações efetivamente realizadas. }\end{array}$ & $\begin{array}{l}\text { Pesquisa qualita- } \\
\text { tiva com aborda- } \\
\text { gem exploratória } \\
\text { e descritiva. }\end{array}$ & $\begin{array}{l}\text { Português } \\
\text { Inglês }\end{array}$ \\
\hline $\begin{array}{l}\text { Patrocinio; Tor- } \\
\text { res; Guariento } \\
\text { (2013) } \\
\text { SciELO }\end{array}$ & $\begin{array}{l}\text { Campinas } \\
- \text { SP. }\end{array}$ & $\begin{array}{l}\text { Avaliar a proposta de um programa de educação popular em saúde para } \\
\text { um envelhecimento saudável em duas comunidades da cidade de Campi- } \\
\text { nas, SP e verificar a influência deste programa sobre os hábitos de vida e } \\
\text { em sintomas depressivos dos participantes. }\end{array}$ & $\begin{array}{l}\text { Pesquisa quan- } \\
\text { titativa. Estudo } \\
\text { exploratório, pros- } \\
\text { pectivo e inter- } \\
\text { vencionista. } \\
\end{array}$ & Português \\
\hline $\begin{array}{l}\text { Coutinho et al. } \\
\text { (2013) } \\
\text { SciELO }\end{array}$ & $\begin{array}{l}\text { Botucatu } \\
- \text { SP }\end{array}$ & $\begin{array}{l}\text { Compreender como vem sendo realizado o cuidado ao idoso em Unidade } \\
\text { de Saúde da Família segundo a visão dos profissionais de saúde. }\end{array}$ & $\begin{array}{l}\text { Pesquisa qualitati- } \\
\text { va, a análise feno- } \\
\text { menológica social } \\
\text { de Alfred Schutz. }\end{array}$ & \\
\hline $\begin{array}{l}\text { Patrocinio; Perei- } \\
\text { ra (2013) } \\
\text { SciELO }\end{array}$ & $\begin{array}{l}\text { Campinas } \\
-\mathrm{SP}\end{array}$ & $\begin{array}{l}\text { Avaliar os efeitos de um programa de educação popular em saúde dirigido } \\
\text { a idosos comunitários sobre suas atitudes em relação à velhice. }\end{array}$ & $\begin{array}{l}\text { Pesquisa quanti- } \\
\text { tativa }\end{array}$ & Português \\
\hline $\begin{array}{l}\text { Valer et al. } \\
(2015) \\
\text { SciELO }\end{array}$ & $\begin{array}{l}\text { Porto Ale- } \\
\text { gre-RS }\end{array}$ & $\begin{array}{l}\text { Descrever o significado de envelhecimento saudável para pessoas idosas } \\
\text { que participam de grupos de educação em saúde de uma Unidade Básica } \\
\text { de Porto Alegre-RS. }\end{array}$ & $\begin{array}{l}\text { Descritivo, de ca- } \\
\text { ráter qualitativo. }\end{array}$ & $\begin{array}{l}\text { Português } \\
\text { Inglês }\end{array}$ \\
\hline $\begin{array}{l}\text { Goes; Polaro; } \\
\text { Gonçalves (2016) } \\
\text { BDENF }\end{array}$ & Pará & $\begin{array}{l}\text { Realizar avaliação diagnóstica de condições de vida e saúde dos idosos } \\
\text { convivendo em família e comunidade, usuários de uma Unidade Básica de } \\
\text { Saúde - UBS - e testar o desenvolvimento de uma tecnologia cuidativo-e- } \\
\text { ducacional. }\end{array}$ & $\begin{array}{l}\text { Método Misto. } \\
\text { Pesquisa conver- } \\
\text { gente assistencial. }\end{array}$ & Português \\
\hline $\begin{array}{l}\text { Costa et al., } \\
(2016) \\
\text { SciELO }\end{array}$ & Belém - PA & $\begin{array}{l}\text { Avaliar pertinência e efetividade da tecnologia cuidativo-educacional } \\
\text { "contação de histórias" como estratégia no cultivo do envelhecimento } \\
\text { ativo (EA) para usuários idosos de uma Unidade Básica de Saúde (UBS), da } \\
\text { Amazônia. }\end{array}$ & $\begin{array}{l}\text { Pesquisa qualitati- } \\
\text { va, do tipo conver- } \\
\text { gente assistencial. }\end{array}$ & $\begin{array}{l}\text { Português } \\
\text { Inglês }\end{array}$ \\
\hline $\begin{array}{l}\text { Sá; Cury; Ribeiro } \\
\text { (2016) } \\
\text { SciELO }\end{array}$ & $\begin{array}{l}\text { Diamantina } \\
-M G\end{array}$ & $\begin{array}{l}\text { Identificar e descrever as ações de promoção à saúde relacionadas à } \\
\text { atividade física de idosos nas unidades básicas de saúde, assim como a } \\
\text { percepção dos responsáveis sobre essa prática. }\end{array}$ & $\begin{array}{l}\text { Pesquisa quanti- } \\
\text { tativa. }\end{array}$ & $\begin{array}{l}\text { Português } \\
\text { Inglês }\end{array}$ \\
\hline
\end{tabular}

Fonte: Elaboração própria do autor. Recife-PE, 2017. 
de vida como boa ou muito boa. Além disso, os autores indicam a avaliação da qualidade de vida como fator essencial para orientação de práticas de saúde efetivas direcionadas a essa população. Por outro lado, Valer et al. (2015), ao analisarem o significado de envelhecimento saudável entre idosos participantes de grupos de educação em saúde, identificaram que tais indivíduos visualizam essa temática de forma ampliada, associando-a a questões relacionadas a comportamentos saudáveis, ter uma rede de apoio, autonomia, pensamentos positivos, entre outros.

\section{Percepção dos profissionais sobre as ações de educação em saúde}

De maneira geral, os estudos mostram que, na perspectiva da atenção básica, as ações de educação em saúde são reconhecidas como essenciais para o cuidado destinado à pessoa idosa, no sentido de garantir aos sujeitos empoderamento relacionado a sua saúde. Dificuldades, contudo, são apontadas pelos profissionais no que diz respeito à concretização de ações, tais como a baixa assiduidade dos idosos na unidade de saúde, necessidade de capacitação dos profissionais para melhor atender este público e de sensibilização dos gestores para a criação de redes de cuidado que incluam a pessoa idosa e, ainda, a falta de insumos para dar suporte às atividades educativas (BARROS; MAIA; PAGLIUCA, 2011; ARAÚJO et al., 2013; COUTINHO et al., 2013; ARAÚJO; BARBOSA, 2010).

Salienta-se que, além dos profissionais de nível superior, os agentes comunitários de saúde também se configuram como essenciais para a realização de ações educativas, muito embora o estudo tenha demonstrado que tais profissionais não detêm conhecimentos satisfatórios acerca do envelhecimento (CRUZ; MARTINS, 2010).

Outro estudo evidencia que os profissionais consideram a comunicação um fator essencial para a operacionalização de atividades educativas, embora os autores chamem atenção para a utilização indevida de práticas tradicionais de ensino, também conhecidas como bancárias, nas quais as informações são simplesmente transferidas de profissionais para usuários. Deste modo, cabe aos profissionais selecionar os momentos oportunos para difundir conhecimentos, utilizando sempre linguagem clara, simples e objetiva (ARAÚJO; BARBOSA, 2010).

\section{Estratégias de educação em saúde para idosos}

Verificou-se na literatura que as ações de educação em saúde devem ser centradas nos usuários dos serviços, tornando-se essencial verificar, a partir desses indivíduos, as suas angústias e necessidades, na tentativa de construir atividades educativas que possam ter significado na vida dos idosos.

Em sua pesquisa convergente-assistencial, Goes, Polaro e Gonçalves (2016) desenvolveram uma tecnologia "cuidativo-educacional" para idosos de uma unidade de saúde, na qual eram debatidos com os idosos assuntos que são do seu interesse. Tal instrumento mostrou-se eficaz no que se diz respeito ao desenvolvimento de habilidades para o autocuidado, uma vez que, nos encontros para discussão em grupo, os envolvidos puderam trocar experiências e vivências.

Grupos de educação destinados a indivíduos com doenças específicas também foram citados na literatura, mais especificamente os relacionados ao cuidado com a Hipertensão Arterial. Em estudo de González (2008) verifica-se como se processa a dinâmica no interior desses grupos. Determinados profissionais indicam a real participação dos usuários em todo o processo educativo, ao mesmo tempo em que outros revelam um posicionamento mais rígido no tocante à elaboração de tais momentos.

Por outro lado, Baldissera e Bueno (2012) trazem, em seu artigo, uma proposta para trabalhar a sexualidade entre idosas participantes de um grupo de hipertensas. As autoras dividiram esse processo em três etapas, no qual, nas duas primeiras, por meio de grupo focal e entrevistas, verificaram as percepções das idosas a respeito da temática e selecionaram propostas para serem trabalhadas no terceiro momento, que se consistiu em quatro encontros, nos quais os temas geradores eram debatidos utilizando grupos focais, painéis dialogados, fotografias, entre outros métodos. Salienta-se que tal processo passou por avaliações formativas e somativas, no intuito de verificar os sentimentos das mulheres diante das experiências.

Outra interessante experiência, desenvolvida por Costa et al. (2016), teve por objetivo avaliar uma tecnologia "cuidativo-educacional" com idosos conhecida como "contação de histórias". Nessa estratégia as idosas passaram por processos educativos para o desenvolvimento de habilidades expressivas. As autoras verificaram que essa tecnologia relacional permitiu o estímulo à memória das idosas, a interação social e a convivência em grupo.

Dois estudos tiveram como referencial teórico as pesquisas de Paulo Freire para uma educação libertadora. Em seu artigo, Patrocinio, Torres e Guariento (2013) aplicaram um programa de educação popular com idosos sobre envelhecimento saudável, baseado no modelo de educação dialógica do famoso au- 
tor, que leva em consideração aspectos relacionados à saúde, participação social, proteção e treinamento contínuo. Tais assuntos eram discutidos em reuniões semanais que duravam, em média, 150 minutos. Por outro lado, Baldissera e Bueno (2012) desenvolveram atividades educativas levando em consideração o entendimento dos idosos sobre lazer. Como referencial teórico utilizaram a pedagogia critico-social de Freire. Nesse estudo, os idosos compreenderam o lazer como estratégia para enfrentar a solidão, a independência, a socialização e a saúde mental. A partir disto, foi posta em prática uma estratégia educativa pautada em dinâmicas e grupos de discussão, que permitiram a troca de experiências e vivências entre os participantes.

Por fim, Sá, Cury e Ribeiro (2016) investigaram as principais ações educativas de promoção à saúde relacionadas a atividades físicas desenvolvidas em uma unidade básica de saúde. Os autores verificaram que as ações mais frequentes foram grupos de caminhada e alongamento, grupo de coluna e Qigong.

\section{Avaliação das ações de educação em saúde na qualidade de vida de idosos}

Verificou-se na literatura que ações educativas se configuram como ferramentas positivas no que diz respeito ao potencial que possuem para modificar hábitos de vida, culminando em aumento das práticas de exercícios físicos, consumo de alimentos saudáveis e no entendimento da velhice como algo positivo (PATROCINIO; TORRES; GUARIENTO, 2013; PATROCINIO; PEREIRA, 2013). Percebeu-se que tais ações também permitem melhoria em aspectos relacionados à saúde mental dos sujeitos, uma vez que comporta o enfrentamento da solidão e garante momentos de socialização e melhorias na autopercepção e autoestima dos idosos (BALDISSERA; BUENO, 2012; COSTA et al., 2016).

\section{DISCUSSÃO}

Semelhante aos artigos que compuseram a amostra do presente estudo, outros autores evidenciam que a percepção do envelhecimento por parte dos idosos está fortemente atrelada a fatores relacionados à autonomia e independência dos indivíduos, hábitos de vida saudáveis, redes de apoio e inclusão social. Apesar de enxergarem o envelhecimento como uma conquista, sentimentos negativos relacionados à necessidade de depender de outras pessoas e deixar de realizar atividades sozinhos, podem fazer parte do imaginário de indivíduos nessa faixa etária (MARINHO et al., 2016; MEDEIROS et al., 2016).
No que diz respeito às dificuldades relacionadas ao processo de formação dos profissionais, principalmente às temáticas recentemente incorporadas ao cotidiano profissional, como o envelhecimento, Weykamp et al. (2016) indicam que a educação permanente dos profissionais no nível de atenção básica é fator crucial para o desenvolvimento de habilidades e competências que possam ser direcionadas para as necessidades dos usuários dos serviços de saúde, sendo essa prática necessária para o reconhecimento e valorização desses indivíduos.

Outro estudo também evidencia a necessidade de valorização da Educação Permanente dos profissionais atuantes nesse nível de atenção, no intuito de melhorar as ações de educação em saúde destinadas a idosos, tendo em vista que nos processos de formação acadêmica muitas vezes essas questões são negligenciadas pelos currículos das instituições de grau superior (TSUNODA et al., 2016).

Em estudo desenvolvido no Triângulo Mineiro, verificou-se que os profissionais da atenção básica enxergam como atributos essenciais para o desenvolvimento de ações educativas o conhecimento acerca de dinâmicas de grupo, doenças e agravos prevalentes nessa população, didática e divulgação das atividades (MENDONÇA et al., 2017).

No que concerne ao posicionamento dos profissionais ante as ações educativas, Silva et al. (2015) concordam com os autores que fizeram parte da amostra do presente estudo, uma vez que encontraram em sua pesquisa posicionamentos semelhantes aos mesmos. Os autores evidenciaram que os profissionais da atenção básica entrevistados em seu estudo acreditavam em uma educação em saúde pautada em aspectos da educação participativa, levando em consideração o posicionamento de todos os sujeitos envolvidos, muito embora também tenham verificado profissionais que acreditam em métodos educativos voltados às concepções mais "bancárias" desse processo.

Salienta-se que, tal qual os artigos encontrados nas bases de dados, as ações de educação em saúde devem estar direcionadas para a emancipação dos sujeitos, com vistas ao empoderamento dos mesmos nos aspectos relacionados a sua saúde (SALCl et al., 2013). Em interessante revisão integrativa da literatura, verifica-se que qualidade de vida e envelhecimento saudável são fatores resultantes de estratégias educativas em saúde, achados semelhantes ao do presente estudo (MALLMAN et al., 2015). 


\section{CONSIDERAÇÕES FINAIS}

O presente estudo permitiu verificar, na literatura, que os idosos que participam de ações educativas tendem a avaliar sua saúde de maneira mais positiva, associando a ela questões relacionadas ao convívio em sociedade, autonomia e pensamentos positivos. Por sua vez, os profissionais identificam a educação em saúde como fator necessário para a atenção destinada à população idosa, muito embora identifiquem dificuldades para a execução de tais práticas, como a falta de preparo para lidar com questões relacionadas ao envelhecimento, o descaso dos gestores para com essa temática e a falta de insumos para a realização de práticas educativas.

Verificaram-se inúmeras estratégias educativas relatadas na literatura. Pode-se perceber também preocupação dos pesquisadores no tocante à compreensão dos idosos como sujeitos ativos no processo de educação, devendo esse momento ser orientado por suas necessidades, para que, assim, compreendam uma ação que tenha significado na vida dos mesmos. Verificou-se predominância de estudos preocupados com ações educativas de caráter mais dialógico, nos quais metodologias ativas foram empregadas, uma vez que a participação dos idosos durante a execução das mesmas era de fundamental importância.

Estudos também evidenciaram que a incorporação de ações educativas no cotidiano dos serviços de saúde garante melhorias na qualidade de vida dos idosos e um envelhecimento saudável, tanto nos aspectos biológicos, relacionados à alimentação saudável e à prática de atividades corporais, quanto naqueles relacionados aos aspectos psicossociais, intimamente ligados à saúde mental melhorada, sociabilização e melhoria da autoestima dos idosos.

Notou-se que as ações de educação em saúde, quando incorporadas na realidade da atenção básica, garantem melhorias no tocante ao envelhecimento saudável da população que faz uso desse serviço de saúde. Percebeu-se uma rica produção bibliográfica nos últimos dez anos acerca dessa temática, evidenciando um crescente interesse por investigá-la, podendo estar associado ao aumento da população idosa.

Os achados deste estudo podem orientar as tomadas de decisão dos profissionais que atuam nesse nível de atenção. Assim, orienta-se a utilização da educação em saúde enquanto ferramenta capaz de potencializar a autonomia dos sujeitos perante as suas necessidades de saúde.

\section{REFERÊNCIAS}

ANTONIO, A. C. F. T.; TONHOM, S. F. R.; CHIRELLI, M. Q. Caution the elderly in primary care: education practice in health physical therapist. Rev. Bras. Promoç. Saúde, Fortaleza, v. 29, n. Supl, p. 5-15, dez. 2016.

ARAÚJO, M. A. S.; BARBOSA, M. A. Relação entre o profissional de saúde da família e o idoso. Esc Anna Nery (impr.), Rio de Janeiro, v. 14, n. 4, p. 819-824, out./dez. 2010.

ARAÚJO, V. S. et al. Discourse of the Collective Subject regarding Education of Health in the Aging Process: a descriptive study. On-line Braz $j$ Nurs [Internet], Rio de Janeiro, v. 12 , n. 2, p. 565-73, 2013. Disponível em: <http://www.objnursing.uff.br/index.php/nursing/article/view/4093>. Acesso em: 30 set. 2017.

BALDISSERA, V. D. A.; BUENO, S. M. V. O lazer e a saúde mental das pessoas hipertensas: convergência na educação para a saúde. Rev. Esc. Enferm. USP, São Paulo, v. 46, n. 2, p. 380-387, 2012.

BALDISSERA, V. D. A.; BUENO, S. M. V. A representação da sexualidade por idosas e a educação para a saúde. Rev. Eletr. Enf. [Internet], v. 12, n.4, p. 622-629, out./dez. 2010. Disponível em: <http://dx.doi.org/10.5216/ree.v12i4.8830>. Acesso em: 29 set. 2017.

BALTES, P.; FREUND, A.; LI, S. C. The psychological science of human ageing. In: JOHNSON, M. L. et al. (Ed.). The Cambridge handbook of age and ageing. Cambridge: Cambridge University Press, 2005. p. 47-71.

BARROS, T. B.; MAIA, E. R.; PAGLIUCA, L. M. F. Facilidades e dificuldades na assistência ao idoso na estratégia de saúde da família. Rev. Rene, Fortaleza, v. 12, n. 4, p. 732-741, out./ dez. 2011.

BORGES, G. M.; CAMPOS, M. B.; SILVA, L. G. C. Transição da estrutura etária no Brasil: oportunidades e desafios para a sociedade nas próximas décadas. In: INSTITUTO BRASILEIRO DE GEOGRAFIA E ESTATÍSTICA (IBGE). Mudança demográfica no Brasil no início do século XXI: subsídios para as projeções da população. Rio de Janeiro: IBGE, 2015. p. 138-151.

BRASIL. Ministério da Saúde. Secretaria de Atenção à Saúde. Departamento de Atenção Básica. Envelhecimento $e$ saúde da pessoa idosa. Brasília: Ministério da Saúde, 2006. $192 \mathrm{p}$.

. Ministério da Saúde. Secretaria-Executiva. Secretaria de Gestão do Trabalho e da Educação na Saúde. Glossário temático: gestão do trabalho e da educação na saúde. 2 . ed. Brasília: Ministério da Saúde, 2012. 44 p. (Série A. Normas e Manuais Técnicos).

CARVALHO, M. H. R. et al. Tendência de mortalidade de idosos por doenças crônicas no município de Marília-SP, Brasil: 1998 a 2000 e 2005 a 2007. Epidemiol. Serv. Saúde, Brasília, v. 23, n. 2, p. 347-354, abr./jun. 2014.

COSTA, N. P. et al. Contação de história: tecnologia cuidativa na educação permanente para o envelhecimento ativo. Rev. Bras. Enferm., [Internet], v. 69, n. 6, p. 1.068-1.075, nov./dez. 2016. Disponível em: <http://www.scielo.br/scielo. php?pid=S0034-71672016000601132\&script=sci_abstract\&tlng=pt>. Acesso em: 30 set. 2017. 
CRUZ, A. L. B.; MARTINS, A. K. L. Percepção da promoção da saúde do idoso: olhar de agentes comunitários de saúde. Rev. Enferm. Ufpe [on-line], v. 4, n. 3, p. 1.484-1.491, jul./ set. 2010. Disponível em: <file:///C:/Users/Jaime\%20Rodrigues/Downloads/1056-11620-1-PB\%20(2).pdf>. Acesso em: 29 set. 2017.

COUTINHO, A. T. et al. Integralidade do cuidado com o idoso na estratégia de saúde da família: visão da equipe. Esc Anna Nery (impr.) [on-line], v. 17, n .4, p. 628-637, out./dez. 2013.

FEIJÃO, A. R.; GALVÃO, M. T. G. Ações de educação em saúde na atenção primária: revelando métodos, técnicas e bases teóricas. Rev. Rene, Fortaleza, v. 8, n. 2, p. 41-49, maio/ ago. 2007.

FELKENBERG, M. B. et al. Educação em saúde e educação na saúde: conceitos e implicações para a saúde coletiva. Ciênc. Saúde Coletiva, v. 19, n. 3, p. 847-849, 2014.

FERREIRA, S. L. et al. Qualidade de vida de idosos que participam de grupo de promoção da saúde. Enfermería Global. n. 40, p. 1-11, 2015.

GOES, T. M.; POLARO, S. H. I.; GONÇALVES, L. H. T. Cultivo do bem viver das pessoas idosas e tecnologia cuidativo-educacional de enfermagem. Enferm. Foco, v. 7, n. 2, p. 4751, abr./jun. 2016.

GONZÁLEZ, C. R. A. A promoção da saúde como caminho para o envelhecimento ativo: o cuidado ao hipertenso em um centro de saúde escola. 2008. Dissertação (Mestrado em Saúde Pública) - Programa de Pós-Graduação em Saúde Pública, Escola Nacional de Saúde Pública Sérgio Arouca, Rio de Janeiro, 2008.

INSTITUTO BRASILEIRO DE GEOGRAFIA E ESTATÍSTICA. IBGE. Síntese de indicadores sociais: uma análise das condições de vida da população brasileira: 2015. Rio de Janeiro: IBGE, 2015.

MACIEL, M. E. D. Educação em saúde: conceitos e propósitos. Cogitare Enferm., v. 14, n. 4, p. 773-776, out./dez. 2009.

MALLMAN, D. G. et al. Educação em saúde como principal alternativa para promover a saúde do idoso. Ciência \& Saúde Coletiva, Rio de Janeiro, v. 20, n. 6, p. 1.763-1.772, jun. 2015.

MARINHO, V. T. et al. Percepção de idosos acerca do enveIhecimento ativo. Rev. Enferm. Ufpe [on-line], Recife, v. 10, n. 5, p. 1.571-1.578, maio 2016. Disponível em: <https://periodicos.ufpe.br/revistas/revistaenfermagem/article/viewFile/11151/12663>. Acesso em: 3 out. 2017.

MAROSINI, M. V.; FONSECA, A. F.; PEREIRA, I. B. Educação em saúde. In: PEREIRA, I. B.; LIMA, J. C. F. (Org.). Dicionário da educação profissional em saúde. Rio de Janeiro: EPSJV, 2008. p. 155-161.

MEDEIROS, D. V. et al. A percepção do idoso sobre a velhice. Rev. Enferm. Ufpe [on-line], Recife, v. 10, n. 10, p. 3.8513.859, out. 2016. Disponível em: <http://pesquisa.bvs.br/ aps/resource/pt/bde-30116>. Acesso em: 3 out. 2017.

MENDONÇA, F. T. N. F. et al. Health education with older adults: action research with primary care professionals. Rev. Bras. Enferm. [Internet], v. 70, n. 4, p. 792-799, 2017.
Disponível em: <http://www.scielo.br/pdf/reben/v70n4/ pt_0034-7167-reben-70-04-0792.pdf>. Acesso em: 19 jul. 2018.

OLIVEIRA, T. C.; MEDEIROS, W. R.; LIMA, K. C. Diferenciais de mortalidade por causas nas faixas etárias limítrofes de idosos. Rev. Bras. Geriatr. Gerontol, Rio de Janeiro, v. 18, n. 1, p. 85-94, 2015.

ORGANIZAÇÃO MUNDIAL DA SAÚDE. Relatório mundial de envelhecimento e saúde. Genebra: OMS, 2015.

PATROCINIO, W. P.; PEREIRA, B. P. C. Efeitos da educação em saúde sobre atitudes de idosos e sua contribuição para a educação gerontológica. Trab. Educ. Saúde, Rio de Janeiro, v. 11, n. 2, p. 375-394, maio/ago. 2013.

PATROCINIO, W. P.; TORRES, S. V. S.; GUARIENTO, M. L. Programa de educação popular em saúde: hábitos de vida e sintomas depressivos em idosos. Rev. Bras. Geriatr. Gerontol., Rio de Janeiro, v. 16, n. 4, p. 781-792, 2013.

PEREIRA, D. S.; NOGUEIRA J. A. D.; SILVA, C. A. B. Qualidade de vida e situação de saúde de idosos: um estudo de base populacional no Sertão Central do Ceará. Rev. Bras. Geriatr. Gerontol., Rio de Janeiro, v. 18, n. 4, p. 893-908, 2015.

SÁ, P. H. V. O.; CURY, G. C.; RIBEIRO, L. C. C. Atividade física de idosos e a promoção da saúde nas unidades básicas. Trab. Educ. Saúde, Rio de Janeiro, v. 14, n. 2, p. 545-558, maio/ago. 2016.

SALCI, M. A. et al. Educação em saúde e suas perspectivas teóricas: algumas reflexões. Texto Contexto Enferm., Florianópolis, v. 22, n. 1, p. 224-230, jan./mar. 2013.

SANTOS, L. F. et al. Qualidade de vida de idosos que participam de grupo de promoção da saúde. Rev. Enfermería Global, n. 40, p. 1-11, out. 2015.

SILVA, V. L. Mortalidade do idoso e determinantes sociais: descrição da literatura e caracterização no município do Recife-PE, através do Sistema de Informação sobre Mortalidade. 2012. Tese (Doutorado) - Centro de Pesquisas Aggeu Magalhães, Fundação Oswaldo Cruz, Recife, 2012.

SILVA, J. R. A. et al. Educação em saúde na estratégia de saúde da família: percepção dos profissionais. Rev. Bras. Promoção da Saúde, Fortaleza, v. 28, n. 1, p. 75-81, jan./ mar. 2015.

TSUNODA, A.C.F. et al. Cuidado ao idoso na Atenção Básica: práticas de educação em saúde do fisioterapeuta. Revista Brasileira de Promoção da Saúde, v. 29, p. 5-15, 2016.

URSI, E. S. Prevenção de lesões de pele no perioperatório: revisão integrativa da literatura. Ribeirão Preto. 2005. Dissertação (Mestrado) - Universidade de São Paulo, Escola de Enfermagem de Ribeirão Preto, 2005.

VALER, D. B. et al. O significado de envelhecimento saudável para pessoas idosas vinculadas a grupos educativos. Rev. Bras. Geriatr. Gerontol., Rio de Janeiro, v. 18, n. 4, p. 809-819, 2015.

WEYKAMP, J. M. et al. Educação permanente em saúde na atenção básica: percepção dos profissionais de enfermagem. Rev. Enferm. UFSM, Santa Maria, v. 6, n. 2, p. 281-289, abr./jun. 2016. 